\title{
Lehrbuch und Atlas Hüftarthroskopie
}

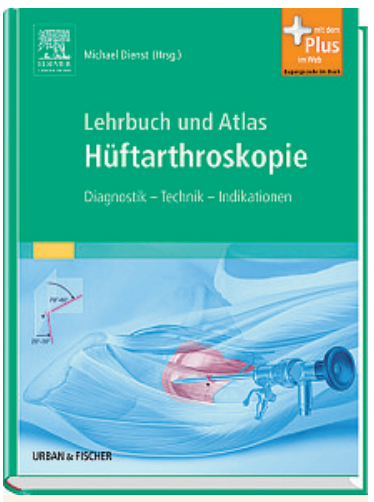

Michael Dienst (Hrsg.) Lehrbuch und Atlas Hüftarthroskopie

München: Urban \& Fischer bei Elsevier; 2009.

444 Seiten, ca. 800 farbige Abb. u. Zeichnungen.

318 CHF.

ISBN 978-3-437-24610-4
Der Herausgeber Dr. med. Michael Dienst ist Privatdozent an der Klinik für Orthopädie des Universitätsklinikums des Saarlandes und Veranstalter und Leiter des alle zwei Jahre stattfindenden «Hip Arthroscopy Meeting» in Homburg/Saar. Es gelang ihm, in der Hüftarthroskopie namhafte Kollegen aus dem In- und Ausland zur Mitarbeit an dem Buch zu gewinnen. Die Anfänge der Hüftarthroskopie reichen noch keine 80 Jahre zurück. Die Entwicklung verlief ähnlich wie bei den Knie- und Schulterarthroskopien. Während noch bis vor wenigen Jahren nur diagnostische Arthroskopien durchgeführt wurden, sind mittlerweile sogar komplexe arthroskopische Operationsverfahren den offenen Verfahren überlegen.

Der Aufbau des Buches ist dreigeteilt und sehr übersichtlich. Bei den allgemeinen Grundlagen finden sich Informationen zu Geschichte, präoperativer Diagnostik, technischen Grundlagen inklusive Lagerung, sowie zu Instrumenten und Zugängen. Der zweite Teil ist der diagnostischen Arthroskopie gewidmet. Anschaulich bebildert und gut beschrieben werden die Zugänge, Kompartimente mit ihrer normalen Anatomie, Variationen und pathologischen Befunde. Im dritten Abschnitt werden die einzelnen Eingriffe erklärt und Kontraindikationen, Komplikationen und die post- operative Rehabilitation behandelt. Den grössten Platz nehmen die Indikationen ein, was verdeutlicht, dass eine operative Methode mit der sorgfältigen Indikationsstellung an Qualität gewinnt.

Das Buch stellt das Gebiet der Hüftarthroskopie auf dem neuesten Stand der Wissenschaft umfassend dar dies erstmalig auf Deutsch.

Auch Nicht-Spezialisten der Hüftarthroskopie werden die Grundlagen vermittelt, indem vermeintlich einfache Aspekte einschliesslich Lagerung, Instrumentengebrauch, arthroskopische Orientierung und Triangulation, Flüssigkeitsmanagement und Blutstillung nochmals aufgegriffen werden. Die bedeutenden Techniken der Hüftarthroskopie, wie zum Beispiel die Portalanlage, werden schrittweise beschrieben. Dabei wird auf die lokale Topographie und Nähe zu neurovaskulären Strukturen hingewiesen. Das PLUS im Internet ist leider noch nicht aktuell. Dort ist lediglich der Zugang zum Roche-Lexikon Medizin zu finden. Ausgewählte Film-Mitschnitte aus dem Bereich Hüftarthroskopie, die in dem Buch angepriesen werden, sucht man leider vergeblich. Aber die Online-Angebote sollten ständig aktualisiert und erweitert werden.

Dr. med. Sandra Krüger, Berlin 